\title{
Acute Respiratory Distress Syndrome
}

\author{
Fawaz Ahmad and Lauren Koffman
}

\subsection{Introduction}

In 1967, Ashbaugh and colleagues first described a respiratory distress syndrome in 12 patients with acute onset of tachypnea, refractory hypoxemia, loss of lung compliance, and diffuse alveolar infiltrates on chest X-ray (CXR) [1]. They noted that these patients had features similar to infantile respiratory distress syndrome, and that they did not respond to standard treatment. Over the years, many different definitions for Acute Respiratory Distress Syndrome (ARDS) have been proposed, with the American European Consensus Conference (AECC) most commonly used in the past and the Berlin definition (Table 26.1) being the most commonly used presently. The Berlin definition of ARDS was published in 2012 and was endorsed by the European Society of Intensive Care Medicine (ESICM), the American Thoracic Society (ATS), and the Society of Critical Care Medicine (SCCM) [2]. They defined ARDS as new or worsening respiratory symptoms within 1 week of clinical insult with bilateral opacities on CXR (Fig. 26.1) or computerized tomography (CT) scan (Fig. 26.2), not fully explained by cardiac failure or fluid overload. They further classified ARDS into three categories (Table 26.2) based on the arterial oxygen tension: fractional inspired oxygen ratio $\left(\mathrm{PaO}_{2} / \mathrm{FiO}_{2}\right)$ and with a minimum positive end-expiratory pressure (PEEP) of 5. The Berlin definition is notably different from prior definitions in that it recognizes that a CT scan can be used in lieu of a chest radiograph, acknowledges that diagnosis can occur in the

Table 26.1 ARDS definition comparisons

\begin{tabular}{l|l|l}
\hline & AECC & Berlin \\
\hline Onset & Acute & $\begin{array}{l}\text { Within a week of known clinical insult or new/ } \\
\text { worsening respiratory symptoms }\end{array}$ \\
\hline Imaging & Bilateral infiltrates on $\mathrm{CXR}$ & Bilateral infiltrates on CXR or CT scan \\
\hline Oxygenation & $\begin{array}{l}\mathrm{ALI}-\mathrm{PaO}_{2} / \mathrm{FiO}_{2} \leq 300 \mathrm{mmHg} \\
\mathrm{ARDS}-\mathrm{PaO}_{2} / \mathrm{FiO}_{2} \leq 200 \mathrm{mmHg}\end{array}$ & $\begin{array}{l}\text { Divided into mild, moderate, and severe as described } \\
\text { in Table } 26.2\end{array}$ \\
\hline PAWP & $\begin{array}{l}<18 \mathrm{mmHg} \text { or no clinical evidence of left } \\
\text { atrial hypertension }\end{array}$ & None \\
\hline
\end{tabular}

F. Ahmad $(\bowtie) \cdot$ L. Koffman

Department of Neurological Sciences, Rush

University Medical Center, Chicago, IL, USA

e-mail: fawaz_ahmad@rush.edu; Lauren_koffman@

rush.edu 


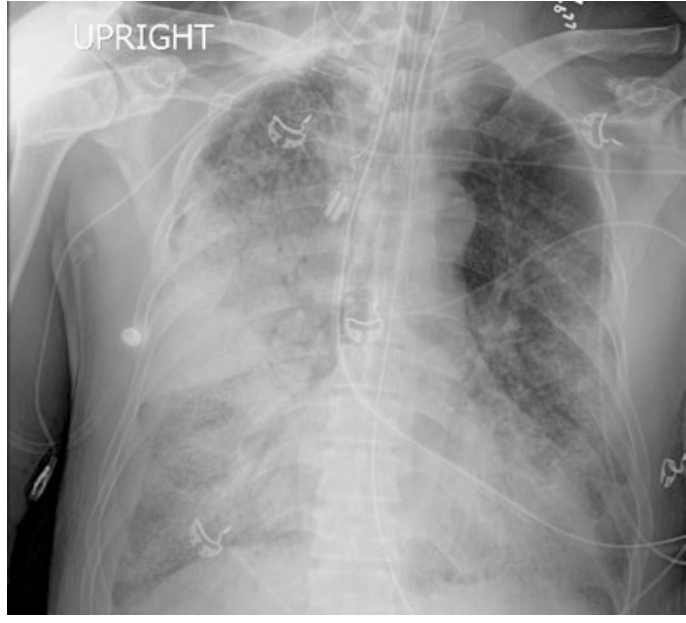

Fig. 26.1 Chest X-ray of a patient showing bilateral lung opacities consistent with ARDS

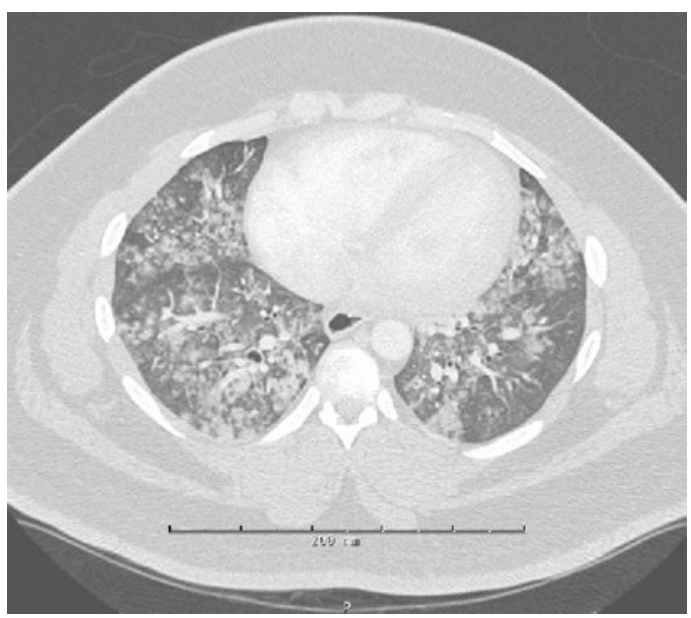

Fig. 26.2 CT chest of a patient with ARDS showing diffuse extensive bilateral focal alveolar airspace opacities

Table 26.2 Berlin definition categories for Acute Respiratory Distress Syndrome (ARDS)

\begin{tabular}{l|l}
\hline Mild & $\begin{array}{l}\mathrm{PaO}_{2} / \mathrm{FiO}_{2} \text { ratio } 201-300 \mathrm{mmHg} \text { with } \\
\\
\mathrm{PEEP} \text { or } \mathrm{CPAP} \geq 5 \mathrm{~cm} \mathrm{H}_{2} \mathrm{O}\end{array}$ \\
\hline Moderate & $\begin{array}{l}\mathrm{PaO} / \\
\mathrm{FiO}\end{array}$ ratio $101-200 \mathrm{mmHg}$ with \\
& $\mathrm{PEEP} \geq 5 \mathrm{~cm} \mathrm{H}_{2} \mathrm{O}$ \\
\hline Severe & $\begin{array}{l}\mathrm{PaO} / \mathrm{FiO}_{2} \text { ratio } \leq 100 \mathrm{mmHg} \text { with PEEP } \\
\geq 5 \mathrm{~cm} \mathrm{H}_{2} \mathrm{O}\end{array}$ \\
\hline
\end{tabular}

presence of cardiac failure, and includes a minimum PEEP of $5 \mathrm{~cm} \mathrm{H}_{2} \mathrm{O}$ (or CPAP for mild ARDS). The Berlin definition simplifies the prior definition by removing the classification of acute lung injury (ALI) and instead utilizing mild, moderate, and severe ARDS classifications. These classifications remain important to the definition of ARDS due to differences in mortality, as well as treatment options.

\subsection{Epidemiology}

Many studies have investigated the incidence of ARDS in acute respiratory failure. An internal report of the National Heart and Lung Institute from 1972 revealed an incidence of ARDS in the USA of 75/100,000 population/year. The Acute Lung Injury: Epidemiology and Natural history (ALIEN) study evaluated patient data from Spain and estimated an incidence of 7.2/100,000 population/year and an ICU mortality of $42 \%$ despite lung protective ventilation [3]. A broader study, which looked at 459 ICUs across 50 different countries, concluded that ARDS represented $10.4 \%$ of total ICU admissions and $23.4 \%$ of all patients on mechanical ventilation [4]. They also noted that ARDS continues to be underdiagnosed, with only $60 \%$ of patients qualifying for ARDS under the Berlin definition being recognized by clinicians.

Risk factors for ARDS include both direct and indirect lung injury. Direct lung injury can occur with pneumonia (bacterial, viral, fungal, and opportunistic), gastric content aspiration, pulmonary contusion, inhalation injury, and drowning. Indirect lung injury includes sepsis, trauma, pancreatitis, burns, drug overdose, and transfusionrelated acute lung injury (TRALI) [2]. The majority of ARDS cases are related to pneumonia, gastric aspiration, and sepsis [5].

Aside from lung injury, it has been hypothesized that genetic predisposition may increase the risk for developing ARDS. Although no single gene has been identified as a culprit, over 40 genes have been associated with ARDS. These include angiotensinconverting enzyme (ACE), epidermal growth factor 
(EGF), factor V, IL6, IL8, IL10, surfactant protein B, tumor necrosis factor-alpha (TNF), and Toll-like receptor 1 (TLR1) [6] among others. ACE has the clearest association with ARDS, which came into light when ACE2 was identified in in vitro studies as a potential receptor for the coronavirus that caused SARS [7]. This was confirmed by showing that ACE2 expression in cells that initially were not susceptible to the SARS infection would allow entry of the virus into the cell [8]. This has led to the possibility of recombinant ACE2 protein as a potential treatment for preventing the spread of SARS and protecting infected patients from worsening lung failure. Studies are also being conducted to better define the role of ACE2 in other emerging lung infections such as avian influenza $\mathrm{A}$.

\subsection{Pathophysiology}

ARDS consists of three distinct phases: the exudative phase, the proliferative phase, and the fibrotic phase. The exudative phase occurs within 1-7 days after the initial insult to the lung. The initial insult may be a direct insult to the alveolar epithelium (pneumonia) or an indirect insult such as pancreatitis. This phase is characterized by immune cell-mediated damage of the alveolar capillary endothelial cells and alveolar epithelial cells. The result is a loss of the alveolar barrier and accumulation of protein-rich edema with the formation of a protein-rich hyaline membrane. Alveolar M1 macrophages secrete cytokines (IL$1,6,8$, and 10), tumor necrosis factor-alpha, and lipid mediators. In response to these proinflammatory cytokines, neutrophils are recruited to the area [9] as well as activation of alveolar epithelial cells and effector T-cells, which leads to sustained inflammation and tissue injury [10]. The resulting inflammatory exudate directly affects type II alveolar cells, leading to inactivation of surfactant and impairment of alveolar function. This cascade of injuries and decreased pulmonary compliance leads to worsening gas exchange, increasing ventilation-perfusion mismatch, and refractory hypoxia which can be further worsened by mechanical stretch injury.
The proliferative phase of ARDS occurs from day 7 to 21 and attempts to repair the damages from the exudative phase. This occurs as M1 macrophages transition into M2 macrophages and help clear debris from the inflammatory process. This also leads to signaling type II alveolar cells which stimulate fibrin matrix scaffolds, synthesize surfactant, and differentiate into type I alveolar cells. As the epithelial lining recovers, protein channels and tight junctions are reestablished, which helps remove alveolar edema.

The final phase of ARDS, the fibrotic phase, does not consistently occur in all patients. This phase consists of extensive alveolar duct and interstitial fibrosis, and emphysema-like changes can be observed. Destruction of pulmonary vasculature and fibrosis leads to increased pulmonary hypertension. This phase is associated with a significant increase in mortality and may require long-term support on mechanical ventilation.

\subsection{Treatment}

Treatment of ARDS consists of identification and treatment of the underlying cause, while minimizing ventilator-induced lung injury and ensuring adequate gas exchange. This method of mechanical ventilation is termed "lung protective ventilation." Noninvasive mechanical ventilation (NIV) is typically not used in patients with ARDS due to the high respiratory failure rates leading to intubation along with the concern for complications associated with delay in mechanical ventilation. A recent meta-analysis showed an intubation rate of up to $86 \%$ and a mortality rate ranging from 15 to $71 \%$ with NIV [11].

\subsubsection{Mechanical Ventilation}

The ARDS Network (ARDSnet) group was a network of 12 clinical sites initiated by the National Heart, Lung, and Blood Institute (NHLBI) and National Institutes of Health (NIH) which encompassed 20 years of research and included 5527 patients. This consortium was established to hasten 
the development of therapy for ARDS, and includes numerous trials evaluating the use of fluids, statins, albuterol, steroids, and nutrition. One of the most influential studies, and one from which much of our current strategy for lung protective ventilation comes from, is the landmark ventilation with lower tidal volumes as compared to traditional tidal volumes for acute lung injury and ARDS (ARMA) study which was published by the ARDSnet group in 2000 [12].

The ARMA study looked at patients who were mechanically ventilated due to either acute lung injury or ARDS. Patients were randomized into receiving the higher tidal volume ventilation of $12 \mathrm{~mL} / \mathrm{kg}$ of ideal body weight with plateau pressures $<50 \mathrm{~cm} \mathrm{H}_{2} \mathrm{O}$ or a low tidal volume ventilation of $6 \mathrm{~mL} / \mathrm{kg}$ of ideal body weight and plateau pressures $<30 \mathrm{~cm} \mathrm{H}_{2} \mathrm{O}$. The study was stopped early due to an $8 \%$ reduction of mortality in the low tidal volume ventilation group $(31 \%$ vs $38.8 \%$ ). This led to the belief that higher tidal volume ventilation can lead to worsening pulmonary edema due to alveolar overdistention, which can cause additional damage to the endothelial and epithelial lining, and promote a proinflammatory cascade [13]. While the high tidal volume group in this study was significantly higher than typically used, this study offers evidence showing that mechanical ventilation with low tidal volumes has a reduction in mortality.

There is also much debate as to which mechanical ventilation mode is best suited for patients with ARDS. However, a Cochrane review of three randomized control trials that included 1089 patients showed that there was no evidence to suggest that there was an advantage of using pressurecontrolled or volume-controlled ventilation [14].

\subsubsection{PEEP}

Ideal PEEP settings in ARDS are also controversial and likely vary by patient. While higher PEEP minimizes dead space and alveolar stretch, it can lead to decreased venous return. However, as the PEEP drops, atelectasis can worsen. It is recommended that a minimum PEEP of $5 \mathrm{~cm}$ $\mathrm{H}_{2} \mathrm{O}$ be used, with the goal of minimizing trauma induced by repetitive opening and closing of the alveoli. A meta-analysis of three randomized controlled trials showed that mortality was increased with lower values of PEEP in patients with moderate to severe ARDS [15].

Multiple methods have been suggested to determine the optimal PEEP. The most common method for titration is based on the $\mathrm{FiO}_{2}$. The LOVS (Lung Open Ventilation to Decrease Mortality in the Acute Respiratory Distress Syndrome) study [16] compared patients who received conventional levels of PEEP to those receiving higher levels. The study did not show any significant difference in all-cause mortality or barotrauma in the two arms; however, the higher PEEP strategy did appear to improve some secondary end points. The ARDSnet protocol includes both a lower PEEP and a higher PEEP strategy, seen in Table 26.3. This is utilized to achieve an oxygenation goal on arterial blood gas $\left(\mathrm{PaO}_{2}\right)$ of $55-80 \mathrm{mmHg}$ or an oxygen saturation $\left(\mathrm{SpO}_{2}\right)$ goal of $88-95 \%$. When setting the ventilator to a lower tidal volume, it is often necessary to allow for permissive hypercapnia, often causing a respiratory acidosis.

Another method of PEEP selection is based on the Positive End-Expiratory Pressure Settings in Adults with Acute Lung Injury and Acute Respiratory Distress Syndrome (ExPress) trial, which increased PEEP in a stepwise manner while maintaining a constant tidal volume and an inspiratory plateau pressure between 28 and $30 \mathrm{~cm} \mathrm{H}_{2} \mathrm{O}$ [17]. Subgroup meta-analysis of the ALVEOLI, ExPress, and LOVS trial suggested a survival benefit of using higher PEEP in patients with a $\mathrm{PaO}_{2} / \mathrm{FiO}_{2}$ ratio $<200$.

Table 26.3 ARDSnet PEEP/FiO ${ }_{2}$ table

\begin{tabular}{|c|c|c|c|c|c|c|c|c|}
\hline \multicolumn{9}{|c|}{ Lower PEEP/Higher $\mathrm{FiO}_{2}$} \\
\hline $\mathrm{FiO}_{2}$ & 0.3 & 0.4 & 0.4 & 0.5 & 0.5 & 0.6 & 0.7 & 0.7 \\
\hline PEEP & 5 & 5 & 8 & 8 & 10 & 10 & 10 & 12 \\
\hline $\mathrm{FiO}_{2}$ & 0.7 & 0.8 & 0.9 & 0.9 & 0.9 & 1.0 & & \\
\hline PEEP & 14 & 14 & 14 & 16 & 18 & $18-24$ & & \\
\hline \multicolumn{9}{|c|}{ Higher PEEP/Lower $\mathrm{FiO}_{2}$} \\
\hline $\mathrm{FiO}_{2}$ & 0.3 & 0.3 & 0.3 & 0.3 & 0.3 & 0.4 & 0.4 & 0.5 \\
\hline PEEP & 5 & 8 & 10 & 12 & 14 & 14 & 16 & 16 \\
\hline $\mathrm{FiO}_{2}$ & 0.5 & $0.5-0.8$ & 0.8 & 0.9 & 1.0 & 1.0 & & \\
\hline PEEP & 18 & 20 & 22 & 22 & 22 & 24 & & \\
\hline
\end{tabular}


The Esophageal Pressure-Guided Ventilation (EPVent) trial was conducted to determine the effects of transpulmonary pressure-directed controlled mechanical ventilation in relation to the ARDSnet $6 \mathrm{~mL} / \mathrm{kg}$ tidal volume [18]. Although they weren't explicitly looking at PEEP, their trial resulted in patients within the control arm with higher levels of PEEP and better outcomes. A second EPVent2 trial is currently underway, which is evaluating similar effects against a higher PEEP strategy.

Further confusing the matter is the Alveolar Recruitment for Acute Respiratory Distress Syndrome Trial (ART) [19] published in 2017. This trial enrolled patients with a $\mathrm{PaO}_{2} / \mathrm{FiO}_{2}$ ratio $<200$ and compared a low PEEP strategy (using the same low PEEP table as in the ALVEOLI and LOVS trial) to a stepwise titration in PEEP using recruitment maneuvers. Interestingly, this trial showed a higher rate of mortality in the experimental arm.

\subsubsection{Baby Lung and Driving Pressures}

Quantitative analysis of CT scan images of lungs in patients with ARDS [20] led to the concept of "baby lung." Analysis has shown that ARDS is not homogeneously distributed, but rather consists of portions of lung that is normally aerated and portions that are completely deprived of aeration. This results in a small portion of lung that must fulfill the ventilation requirements of the individual. Research by Amato and colleagues [21] uses this concept to suggest that tidal volumes based on ideal body weight (IBW) is a reflection of the lung volume of healthy individuals and does not take into account that the functional lung of a patient with ARDS is significantly reduced (baby lung). They suggest that taking into account the lower respiratory-system compliance $\left(C_{R S}\right)$ of the functional lung as a ratio of the lung volume based on IBW $\left(\mathrm{V}_{\mathrm{T}}\right)$ provides a better prediction for outcomes. They define this ratio as the driving pressure $\left(\Delta \mathrm{P}=\mathrm{V}_{\mathrm{T}} / \mathrm{C}_{\mathrm{RS}}\right)$, which can be calculated at the bedside as the plateau pressure minus positive end-expiratory pressure
(Pplat - PEEP). They reviewed 3500 patients from nine different trials and concluded that there was a higher mortality rate associated with patients with higher driving pressures. They also noted that the protective benefits of higher PEEP were only seen when it was associated with lower driving pressures. Their analysis suggests that driving pressures above $15 \mathrm{~cm} \mathrm{H}_{2} \mathrm{O}$ may increase mortality. There are ongoing trials evaluating the benefit of reduced driving pressures in ARDS patients.

\subsubsection{Prone Positioning}

The Proning Severe ARDS Patients (PROSEVA) trial [22], a multicenter RCT of 474 patients with severe ARDS $\left(\mathrm{PaO}_{2} / \mathrm{FiO}_{2}<150 \mathrm{~mm} \mathrm{Hg}\right.$ with $\mathrm{FiO}_{2}>0.6$ ), showed that when compared to supine positioning, there was a significant reduction in 28-day mortality with prone positioning ( $16 \%$ in prone vs $32 \%$ in supine). The trial utilized prone positioning for a minimum of 16 consecutive hours per day. Placing a patient in prone position reduces the pleural pressure gradient from nondependent to dependent regions and allows for a more homogeneous distribution of aeration and strain from dorsal to ventral areas of the lung, thus protecting the lung from ventilatorinduced lung injury (VILI) and allowing for improved VQ matching. Absolute contraindications to prone positioning include, but are not limited to: pregnancy, spinal instability, increased intracranial pressure, shock, anterior burns, and chest tubes. Hemodynamic instability (MAP $<65 \mathrm{mmHg}$ ) may be considered a relative contraindication.

Although prone positioning has become more common throughout critical care units, challenges still exist in implementation. One of the most important factors for successful implementation remains physician and nurse training in order to familiarize the process. Prone positioning can result in unintended endotracheal extubation. Oxygenation can transiently decrease with proning, in particular during the turning process. This does not reflect a failure in proning and is often a transient phenomenon. Additionally, 
chest wall compliance can be significantly reduced in the prone position, which may be attributed to a decrease in compliance of the rib cage and diaphragmatic component of the chest wall [23].

\subsubsection{Neuromuscular Blockade}

Spontaneous breathing on mechanical ventilation, in particular with severe ARDS, can have negative consequences such as patient-ventilator dyssynchrony and generation of high transpulmonary pressures which may increase the risk of VILI. Use of paralytic agents can improve patient-ventilator synchrony and lower oxygen consumption by respiratory muscles. The ACURASYS trial [24] looked at the effects of cisatracurium, a neuromuscular blocking agent, for $48 \mathrm{~h}$. The trial showed that when compared to placebo, there was significantly lowered adjusted 90-day mortality in the cisatracurium group, increased ventilator-free days, and decreased ICU days. The trial also showed that patients on cisatracurium had less barotrauma and pneumothoraces and had no significant difference of ICU-acquired paresis. Despite these studies, the Society of Critical Care Medicine has not commented on the use of neuromuscular blocking agents in the most recent ARDS guidelines.

\subsubsection{Steroids}

There has been much interest in studying the effects of corticosteroids in ARDS with the hope of reducing the inflammation process. Unfortunately, no study has shown clear evidence of benefit from steroids use. Meduri [25] showed that there may be a reduction in ICU mortality with the use of methylprednisolone in the early phase of ARDS. However, multiple subsequent studies have not been able to demonstrate any evidence supporting the efficacy and use of steroids in ARDS [26, 27]. The ARDSnet steroid study showed an increase in mortality in patients who were started on steroids more than 14 days after the onset of symptoms [26].

\subsubsection{Fluids}

The Fluid and Catheter Treatment Trial in ARDS (FACTT) [28] looked at outcomes in patients with ARDS who were resuscitated with either a conservative or liberal fluid regimen. Although this study did not show a difference in the 60-day mortality rate, patients receiving conservative fluid management had a reduction in the duration of mechanical ventilation and ICU days. Interestingly, this study also looked at the use of central venous pressure and pulmonary capillary wedge pressure (PCWP) for guiding fluid resuscitation. Like many other studies, they concluded that use of a pulmonary artery catheter did not improve outcomes.

\subsubsection{ECMO}

There has been great interest in utilizing venovenous extracorporeal membrane oxygenation (VV-ECMO) for patients with severe respiratory failure. VV-ECMO is a mode of ECMO that specifically provides support for the lungs by oxygenating blood and removing carbon dioxide externally. The efficacy and economic assessment of conventional ventilatory support versus extracorporeal membrane oxygenation for severe adult respiratory failure (CESAR) trial [29] looked at the utility of transferring patients with severe respiratory failure with a Murray score $\geq 3$ or uncompensated hypercapnia with a $\mathrm{pH}<7.2$ to an ECMO center. This study showed that when compared to conventional ventilator management, 6-month survival without severe disability was higher in patients who were transferred to an ECMO center (63\% vs. $47 \%$ ) but it did not show an increase in survival for those who received ECMO compared to those that did not. At the time of publication, there was insufficient evidence to make guideline recommendations for the use of ECMO in patients with ARDS.

\subsubsection{Nutrition}

Nutrition plays a vital role in the recovery of any critically ill patient. Aside from the obvious benefits of nutrition, enteral feeding has the advantages 
of continuously stimulating the gut and reducing the risk of infection by bacterial translocation. The EDEN trial [30] conducted by the ARDSnet group looked at the difference between trophic and full enteral feeding for the first 6 days of treatment, after which all patients received full enteral feeding. Trophic, or trickle, feeding was defined by a rate of $20 \mathrm{kcal} / \mathrm{h}$, whereas full enteral feeding was defined by a rate of $80 \mathrm{kcal} / \mathrm{h}$. This study showed that there was no difference in outcomes (ventilator-free days, 60-day mortality, and infections) between the two groups. However, it was noted that the full enteral feeding group had higher incidences of gastrointestinal intolerance, resulting in emesis and higher gastric residuals. Patients enrolled in the EDEN trial were also initially concurrently enrolled in the OMEGA trial [31], looking at the benefits of omega 3-fatty acids. However, this trial was stopped early due to futility with interim analysis showing a $10 \%$ absolute increase in mortality in the treatment group ( $26.6 \%$ vs. $16.3 \%)$.

\subsubsection{Other Treatment Considerations}

There have been many studies looking at other treatment modalities in ARDS that have not shown to have much benefit or may cause harm. Once such modality is high-frequency oscillatory ventilation (HFOV). As more research has emerged regarding increased damage to the lung from overstretching or repetitive collapse of the alveoli, the thought was that patients may benefit from the extremely low tidal volumes provided by HFOV (1-2 $\mathrm{mL} / \mathrm{kg})$ at very high rates. A Cochrane review of 10 randomized controlled trials of HFOV in patients with moderate to severe ARDS [32] concluded that the use of highfrequency oscillation did not show any significant difference in hospital or 30-day mortality. One of the trials included in the review was terminated early after multiple in-trial analysis showed that the use of HFOV increased the mortality rate [33].

Inhaled nitric oxide (iNO) is a potent pulmonary vasodilator that is used in the treatment of pulmonary hypertension and was thought to improve oxygenation in patients with ARDS by improving blood flow to the healthy portion of lungs. Unfortunately, multiple trials, as summarized by a Cochrane review [34], did not show any evidence for improved survival with the use of iNO and exposed the patient to adverse effects such as hypotension and renal failure.

The Albuterol for the Treatment of ALI (ALTA) trial conducted by the ARDSnet group looked at the use of aerosolized albuterol [35]. Because beta- 2 agonists had been shown to have anti-inflammatory effects in the lungs of experimental ALI, it was anticipated that the severity of lung injury would be reduced due to a reduction of permeability-induced lung injury. However, this trial was terminated early due to futility after showing no benefit in any of its end points. Similarly, another trial, the $\beta$-Agonist Lung Injury Trial (BALTI-II), was terminated early when interim analysis showed increased 28-day mortality [36].

While no broad clinical guidelines have been established regarding the treatment of ARDS, the ESICM, ATS, and SCCM did release a practice guideline statement in May, 2017 addressing mechanical ventilation in adult patients with ARDS [37]. Though limited, they strongly recommended the use of lung protective ventilation strategies utilizing low tidal volume and low plateau pressures and the use of prone positioning for $>12 \mathrm{~h} /$ day in severe ARDS. Additionally, they recommended consideration for the use of higher PEEP and recruitment maneuvers in patients with moderate to severe ARDS. The practice guidelines recommended against the use of highfrequency oscillatory ventilation and were equivocal on the use of ECMO, stating that additional evidence was necessary to make a definitive recommendation.

\subsection{Conclusion}

Acute Respiratory Distress Syndrome is a complicated disease process and, despite advancements in medicine and technology, continues to have a high rate of mortality and utilizes significant resources. Furthermore, early identification 
of patients with the diagnosis of ARDS continues to remain poor. The hallmark of ARDS management continues to be supportive care with treatment of the underlying process and low tidal volume ventilation. Despite numerous studies, few interventions have shown consistent mortality benefit. Therefore, it is important to remember that treatment should be personalized for each patient based on the clinical scenario and degree of disease severity.

\section{Key Points}

- Acute Respiratory Distress Syndrome results from direct or indirect insult to the alveolar epithelium resulting in damage to alveolar capillary cells.

- Acute Respiratory Distress Syndrome remains under-diagnosed and has an ICU mortality of up to $42 \%$.

- The mainstay of treatment in Acute Respiratory Distress Syndrome is supportive care and lung protective mechanical ventilation.

\section{References}

1. Ashbaugh DG, Bigelow DB, Petty TL, Levine BE. Acute respiratory distress in adults. Lancet. 1967;290:319-23.

2. Ferguson ND, Fan E, Camporota L, et al. The Berlin definition of ARDS: an expanded rationale, justification, and supplementary material. Intensive Care Med. 2012;38:1573-82.

3. Villar J, Blanco J, Añón J, et al. The ALIEN study: incidence and outcome of acute respiratory distress syndrome in the era of lung protective ventilation. Intensive Care Med. 2011;37:1932-41.

4. Bellani G, Laffey JG, Pham T, et al. Epidemiology, patterns of care, and mortality for patients with acute respiratory distress syndrome in intensive care units in 50 countries. JAMA. 2016;315:788-800.

5. Zilberberg M, Epstein S. Acute lung injury in the medical ICU: comorbid conditions, age, etiology, and hospital outcome. Am J Respir Crit Care Med. 1998;157:1159-64.

6. Meyer NJ, Christie JD. Genetic heterogeneity and risk of acute respiratory distress syndrome. Semin Respir Crit Care Med. 2013;34:459-74.
7. Kuba K, Imai Y, Rao S, et al. A crucial role of angiotensin converting enzyme 2 (ACE2) in SARS coronavirusinduced lung injury. Nat Med. 2005;11:875-9.

8. Imai Y, Kuba K, Penninger M. Angiotensin-converting enzyme 2 in acute respiratory distress syndrome. Cell Mol Life Sci. 2007;64:2006-12.

9. Martin TR, Pistorese BP, Chi EY, Goodman RB, Matthay MA. Effects of leukotriene B4 in the human lung. Recruitment of neutrophils into the alveolar spaces without a change in protein permeability. J Clin Invest. 1989;84:1609.

10. Aggarwal NR, King LS, D'Alessio FR. Diverse macrophage populations mediate acute lung inflammation and resolution. Am J Physiol Lung Cell Mol Physiol. 2014;306:L709-25.

11. Agarwal R, Aggarwal AN, Gupta D. Role of noninvasive ventilation in acute lung injury/acute respiratory distress syndrome: a proportion meta-analysis. Respir Care. 2010;55:1653-60.

12. The Acute Respiratory Distress Syndrome Network. Ventilation with lower tidal volumes as compared with traditional tidal volumes for acute lung injury and the acute respiratory distress syndrome. N Engl J Med. 2000;342:1301-8.

13. Corbridge TC, Wood LD, Crawford GP, Chudoba MJ, Yanos J, Sznajder JI. Adverse effects of large tidal volume and low PEEP in canine acid aspiration. Am Rev Respir Dis. 1990;142:311-5.

14. Chacko B, Peter JV, Tharyan P, John G, Jeyaseelan L. Pressure-controlled versus volume-controlled ventilation for acute respiratory failure due to acute lung injury (ALI) or acute respiratory distress syndrome (ARDS). Cochrane Database Syst Rev. 2015; 1:CD008807.

15. Fan E, Del Sorbo L, Goligher EC, et al. An official American Thoracic Society/European Society of Intensive Care Medicine/Society of Critical Care Medicine clinical practice guideline: mechanical ventilation in adult patients with acute respiratory distress syndrome. Am J Respir Crit Care Med. 2017; 195:1253-63.

16. Meade MO, Cook DJ, Guyatt GH, et al. Ventilation strategy using low tidal volumes, recruitment maneuvers, and high positive end expiratory pressure for acute lung injury and acute respiratory distress syndrome: a randomized controlled trial. JAMA. 2008;299:637-45.

17. Mercat A, Richard JC, Vielle B, Jaber S, et al. Positive end-expiratory pressure setting in adults with acute lung injury and acute respiratory distress syndrome: a randomized controlled trial. JAMA. 2008;299:646-55.

18. Talmor D, Sarge T, Malhotra A, et al. Mechanical ventilation guided by esophageal pressure in acute lung injury. N Engl J Med. 2008;359:2095-104.

19. ART Trial Investigators. Effect of lung recruitment and titrated positive end-expiratory pressure (PEEP) vs low PEEP on mortality in patients with acute respiratory distress syndrome: a randomized clinical trial. JAMA. 2017;318:1335-45. 
20. Gattinoni L, Mascheroni D, Torresin A, et al. Morphological response to positive end expiratory pressure in acute respiratory failure. Computerized tomography study. Intensive Care Med. 1986;12:137-42.

21. Amato M, Meade M, Slutsky A, et al. Driving pressure and survival in the acute respiratory distress syndrome. N Engl J Med. 2015;372:747-55.

22. Guérin C, Reignier J, Richard JC, et al. Prone positioning in severe acute respiratory distress syndrome. N Engl J Med. 2013;368:2159-68.

23. Pelosi P, Tubiolo D, Mascheroni D, et al. Effects of the prone position on respiratory mechanics and gasexchange during acute lung injury. Am J Respir Crit Care Med. 1998;157:387-93.

24. Papazian L, Forel JM, Gacouin A, et al. Neuromuscular blockers in early acute respiratory distress syndrome. N Engl J Med. 2019;363:1107-16.

25. Meduri GU, Headley AS, Golden E, et al. Effect of prolonged methylprednisolone therapy in unresolving acute respiratory distress syndrome: a randomized controlled trial. JAMA. 1998;280:159-65.

26. The National Heart, Lung, and Blood Institute Acute Respiratory Distress Syndrome (ARDS) Clinical Trials Network. Efficacy and safety of corticosteroids for persistent acute respiratory distress syndrome. $\mathrm{N}$ Engl J Med. 2006;354:1671-84.

27. Agarwal R, Nath A, Aggarwal AN, Gupta D. Do glucocorticoids decrease mortality in acute respiratory distress syndrome? A meta-analysis. Respirology. 2007;12:585-90.

28. The National Heart, Lung, and Blood Institute Acute Respiratory Distress Syndrome (ARDS) Clinical Trials Network. Comparison of two fluidmanagement strategies in acute lung injury. $\mathrm{N}$ Engl $\mathrm{J}$ Med. 2006;354:2564-75.

29. Peek GJ, Mugford M, Tiruvoipati R, CESAR Trial Collaboration, et al. Efficacy and economic assessment of conventional ventilator support versus extracorporeal membrane oxygenation for severe adult respiratory failure (CESAR): a multicenter randomized controlled trial. Lancet. 2009;374:1351-63.

30. The National Heart, Lung, and Blood Institute Acute Respiratory Distress Syndrome (ARDS) Clinical Trials Network. Initial trophic vs full enteral feeding in patients with acute lung injury: the EDEN randomized trial. JAMA. 2012;307:795-803.

31. Rice T, Wheeler A, Thompson BT. Enteral omega-3 fatty acid, $\gamma$-linolenic acid, and antioxidant supplementation in acute lung injury. JAMA. 2011;306:1574-81.

32. Sud S, Sud M, Friedrich JO, et al. High-frequency oscillatory ventilation versus conventional ventilation for acute respiratory distress syndrome. Cochrane Database Syst Rev. 2016;4:CD004085.

33. Ferguson ND, Cook DJ, Guyatt GH, et al. Highfrequency oscillation in early acute respiratory distress syndrome. N Engl J Med. 2013;368:795-805.

34. Gebistorf F, Karam O, Wetterslev J, Afshari A. Inhaled nitric oxide for acute respiratory distress syndrome (ARDS) in children and adults. Cochrane Database Syst Rev. 2016;(6):CD002787.

35. Matthay MA, Brower RG, Carson $S$, et al. Randomized, placebo-controlled clinical trial of an aerosolized beta-2 agonist for treatment of acute lung injury. Am J Respir Crit Care Med. 2011;184:561-8.

36. Gao Smith F, Perkins GD, Gates S, BALTI-2 study investigators, et al. Effect of intravenous $\beta-2$ agonist treatment on clinical outcomes in acute respiratory distress syndrome (BALTI-2): a multicenter, randomized controlled trial. Lancet. 2012;379:229-35.

37. Fan E, Sorbo LD, Goligher EC, Hodgson CL, et al. An official American Thoracic Society/European Society of Intensive Care Medicine/Society of Critical Care Medicine clinical practice guideline: mechanical ventilation in adult patients with acute respiratory distress syndrome. Am J Respir Crit Care Med. 2017;195:1253-63. 